\title{
Buyang Huanwu decoction increases the expression of glutamate transporter-1 and glutamate synthetase in association with PACAP-38 following focal ischemia
}

\author{
WENTING DING* ${ }^{*}$ PENG YU*, WEI LIU, LEQUAN ZHOU, LI GUAN and RUISHAN LIN \\ Department of Physiology, College of Fundamental Medical Science, Guangzhou University of Chinese Medicine, \\ Guangzhou, Guangdong 510006, P.R. China
}

Received March 24, 2015; Accepted May 5, 2015

DOI: $10.3892 /$ br. 2015.478

\begin{abstract}
The neuroprotective role of Buyang Huanwu decoction (BYHWD) in focal ischemia is associated with decreasing glutamate concentration. However, the mechanisms are not fully understood. The present study aimed to explore whether glutamate transporter-1 (GLT-1) and glutamine synthetase (GS) participated in the decreased level of glutamate and whether pituitary adenylate cyclase-activating polypeptide-38 (PACAP-38) was involved in this process. BYHWD was found to significantly upregulate the expression of GLT-1 and GS in the hippocampal CA1 area compared to the ischemia group, with the difference on day 3 being most significant. BYHWD increased the level of PACAP-38, and PACAP-(6-38) (PACAP receptor antagonist) significantly attenuated the effect of BYHWD on GLT-1 and GS, suggesting that PACAP-38 was involved in the upregulation of GLT-1 and GS induced by BYHWD. In addition, as GLT-1 and GS are mainly located in astrocytes, the changes of astrocytes were detected by glial fibrillary acidic protein (GFAP; an astrocytic marker) immunostaining. The results showed that BYHWD inhibited the expression of GFAP compared with the ischemia group,
\end{abstract}

Correspondence to: Dr Wei Liu, Department of Physiology, College of Fundamental Medical Science, Guangzhou University of Chinese Medicine, 232 Waihuan East Road, Guangzhou, Guangdong 510006, P.R. China

E-mail:weiliu1980@yahoo.com

*Contributed equally

Abbreviations: GLT-1, glutamate transporter-1; GS, glutamine synthetase; BYHWD, Buyang Huanwu Decoction; PACAP-38, pituitary adenylate cyclase-activating polypeptide-38; MCAO, middle cerebral artery occlusion; GFAP, glial fibrillary acidic protein; IR, immunoreactivity

Key words: Buyang Huanwu decoction, focal cerebral ischemia, astrocyte, glutamate transporter-1, glutamine synthetase, pituitary adenylate cyclase-activating polypeptide- 38 however, co-administration with PACAP-(6-38), which inhibited the effect of BYHWD on GLT-1 and GS in astrocytes, attenuated this effect, indicating that astrocytes participated in the protective role of BYHWD following focal ischemia. These results provided the evidence for the first time that not only neurons but also astrocytes contribute to the protective role of BYHWD, which opposes previous studies and may be a starting point for traditional medicine.

\section{Introduction}

Buyang Huanwu decoction (BYHWD), a traditional Chinese herbal prescription, has been used for attenuating ischemic injury for $>300$ years (1). The neuroprotective effects of BYHWD following ischemia are involved in multiple pathways, such as reducing the infarct volume (2), decreasing apoptotic cells (3), inhibiting the oxidative stress/nitration stress reaction (4) and stimulating neural proliferation (5). Recent studies showed that BYHWD greatly suppressed the level of glutamate $(6,7)$; however, the potential mechanisms are not fully understood.

Glutamate is the most common excitatory neurotransmitter in the central nervous system. During cerebral ischemia, its level is elevated markedly causing the glutamate-induced neuronal toxicity, which was considered the initial trigger in ischemic injury (8). Glutamate transporter-1 (GLT-1, also known as EAAT2) contributes $>90 \%$ of glutamate transportation (9). GLT-1 upregulation was involved in the protection of ischemic preconditioning (10), postconditioning (11) and certain drugs, such as $\beta$-lactam antibiotics (12). In addition, the glutamate that was transported into astrocytes would be further degraded by glutamine synthetase (GS). Downregulated GS was reported to increase the toxicity of glutamate and induce severe neuronal injury $(13,14)$. Additionally, pituitary adenylate cyclase-activating polypeptide (PACAP), particularly PACAP-38 (the predominant PACAP form in the nervous system), was shown to be involved in focal ischemia by mediating glial glutamate transportation (15). Elevation of intercellular PACAP-38 induced GLT-1 expression. These effects were attenuated in the presence of PACAP-(6-38), a non-specific PACAP antagonist $(15,16)$. 
Glutamate transportation and metabolism are the only ways to decrease the concentration of glutamate; therefore, we speculated that the effect of BYHWD suppressing the glutamate level may be associated with upregulation of GLT-1 and GS. In the present study, the expression of GLT-1 and GS was examined following BYHWD administration in focal ischemia. In addition, whether the effect of BYHWD on GLT-1 and GS was associated with PACAP-38 and whether the astrocytic marker GFAP was influenced were further investigated. The present study provides evidence that can be used to further elucidate astrocytes participating in the protection of BYHWD following focal ischemia.

\section{Materials and methods}

Composition and preparation of BYHWD. BYNWD (Guangdong Provincial Traditional Chinese Medical Hospital, Second Affiliated Hospital of Guangzhou University of Chinese Medicine, Guangzhou, China) is composed of Radix Astragali (Gansu, China), Radix Angelicae Sinensis (Gansu, China), Radix Paeonia Rubra (Sichuan, China), Rhizoma Chuanxiong (Sichuan, China), Semen Persicae (Hebei, China), Flos Carthami (Sichuan, China) and Lumbricus (Guangxi, China). The components were mixed with a ratio of 120:6:4.5:3:3:3:3 (dry weight) (17). To maintain the consistency of the herbal chemical ingredients, all the herbal components were originally obtained from the standard naive sources as stated above with good agriculture practice grade and the drugs were extracted with standard methods according to Chinese Pharmacopoeia (China Pharmacopoeia and Committee, 2000). BYHWD was prepared with distilled water at $100^{\circ} \mathrm{C}$ for 30 min twice and the drug solution was concentrated to the final concentration of $2 \mathrm{~g} / \mathrm{ml}$ (equivalent to dry weight of raw materials).

Animalmodelofcerebralischemia.AdultmaleSprague-Dawley rats weighing 220-250 g were purchased from Guangzhou University of Chinese Medicine Experimental Animal Center (Guangzhou, China). All the experimental procedures were carried out strictly in compliance with the NIH guidelines for the Care and Use of Laboratory Animals. Focal ischemia was induced by middle cerebral artery occlusion (MCAO) as previously reported (18). Briefly, rats were anesthetized with $350 \mathrm{mg} / \mathrm{kg}$ chloral hydrate via intraperitoneal injection. The right carotid bifurcation was exposed, and the external carotid artery was coagulated distal to the bifurcation. A 4-0 nylon monofilament suture with a rounded tip was introduced into the internal carotid artery through the stump of the external carotid artery and gently advanced for $\sim 20 \mathrm{~mm}$. After $2 \mathrm{~h}$ of occlusion, the filament was gently withdrawn, and the incision was closed. During the whole process, the rat rectal temperature was maintained at $37^{\circ} \mathrm{C}$ by placing the animals on a heating bed. In the sham-operated rats, the common carotid was exposed without ligation. Two hours after surgery, the rats were intragastrically administered with BYHWD every $12 \mathrm{~h}$ at the dose that was equivalent to $36 \mathrm{~g}$ raw materials/(kg/day) (1). The other rats were administered the same volume of distilled water. In some experiments, the rats were injected intravenously with the PACAP receptor antagonist PACAP-(6-38) (Tocris Bioscience, Ellisville, MO, USA) every 48 h (19).
Ethical approval. The study was approved by the Ethics Committee of the Guangzhou University of Chinese Medicine (no. ds20130001).

Immunohistochemistry. Following the experiments, the animals were anesthetized with an overdose of $10 \%$ chloral hydrate and perfused through the ascending aorta with $0.9 \%$ saline and $4 \%$ buffered paraformaldehyde. Brains were quickly removed, post-fixed in the same fixative for $2 \mathrm{~h}$ and subsequently transferred into a graded series of phosphate-buffered sucrose solutions (10-30\%) at $4^{\circ} \mathrm{C}$. Coronal sections $(15 \mu \mathrm{m})$ were embeded in optimum cutting temperature compound, and cut at the levels of hippocampus (22.8-23.8 $\mathrm{mm}$ from the bregma) on a cryostat and mounted on polylysine-coated slides. Sections were used to stain individually for the following primary antibodies: Anti-GLT-1 (1:400; cat. no. AB1783; Merch Millipore, Billerica, MA, USA), anti-GS (1:400; cat. no. ab73593; Abcam, Cambridge, MA, USA) and anti-GFAP (1:800; cat. no. MAB360; Merck Millipore) overnight at $4^{\circ} \mathrm{C}$. For immunohistochemistry, the sections were incubated with biotinylated immunoglobulin $\mathrm{G}$ and further processed with the avidin-biotin complex method (Vector, San Diego, CA, USA). For immunofluorescence, the sections were incubated with the corresponding secondary antibodies for $2 \mathrm{~h}$ at room temperature and captured after rinsing. For double immunofluorescence, sections were incubated with a mixture of antibodies, anti-GLT-1, anti-GS and anti-GFAP (astrocyte marker), followed by a mixture of fluorescein isothiocyanate- and CY3-conjugated secondary antibodies (Chemicon, Temecula, CA, USA). Images were captured using an Olympus microscope (Olympus, Tokyo, Japan). To quantify GLT-1, GS and GFAP immunoreactivity (IR), measurement was performed with a computerized image analysis system (Image-Pro-Plus; Media Cybernetics, Silver Spring, MD, USA) according to a previously described method (18). Briefly, a density threshold was first set above the background level to identify the positively stained structure and the area occupied by these structures was measured as the positive area. An average percentage of the immunoreactive area to the total outlined area was obtained. Five to six animals were included in each group for quantification of immunohistochemistry.

Examination of PACAP-38 level. PACAP-38 was measured by the PACAP-38 enzyme-linked immunosorbent assay (ELISA) kit (TSZ Bioscience, North Brunswick, NJ, USA). Briefly, rats were decapitated and their brains were quickly removed. Hippocampi were weighed and immersed in 10 -fold (volume/hippocampus weight) $0.2 \mathrm{~mol} / 1 \mathrm{Tris}-\mathrm{HCl}$ buffer (pH 7.3) containing $20 \mathrm{mmol} / \mathrm{EDTA}$, and subsequently boiled and centrifuged as previously described (20). Relevant reagents in the kit were added according to the manufacturer's instructions. The absorbance of the product was measured at $450 \mathrm{~nm}$ using a microplate ELISA reader (Bio-Rad, Hercules, CA, USA). The concentration of PACAP-38 in the samples was estimated from the standard curve that was constructed in each assay. Samples were assayed in triplicate.

Statistical analysis. Observers blinded to experimental conditions evaluated the outcome measures. All the data 
A

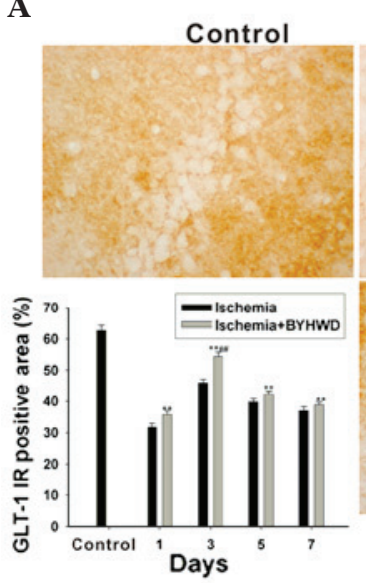

B
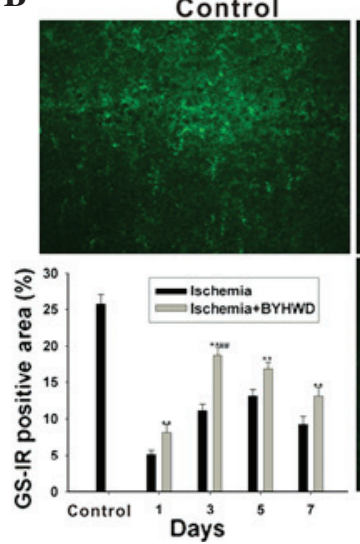

GLT-1
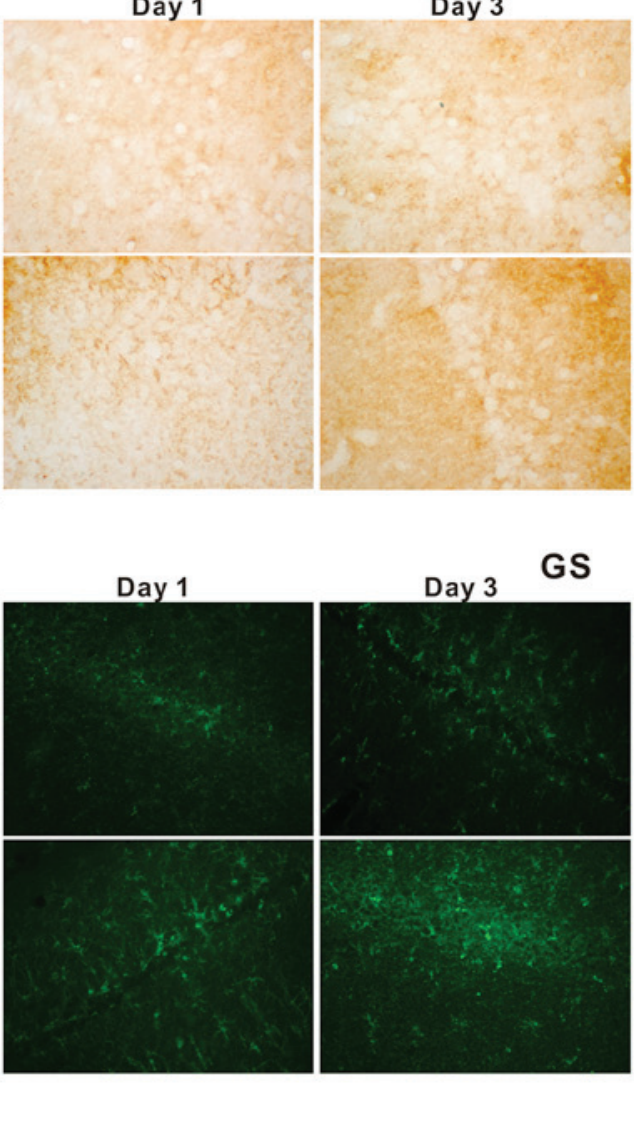

Day 3

GS

Day 5

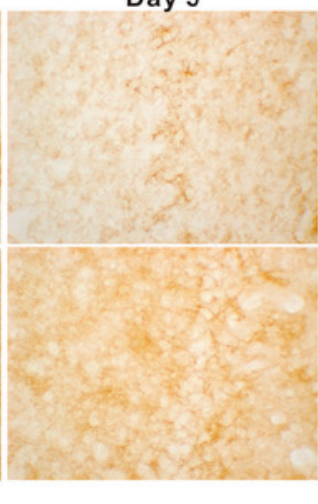

Day 5

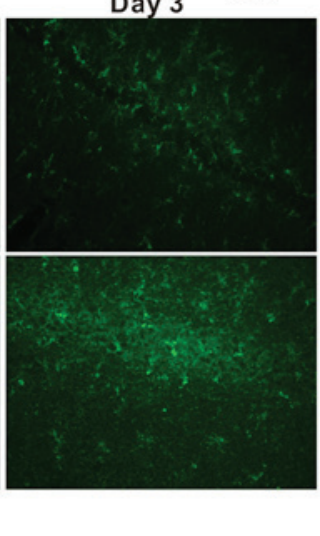

Day 7

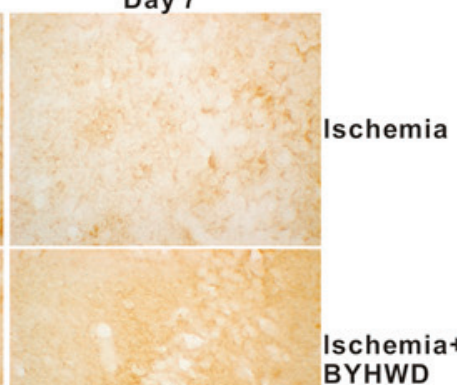

BYHWD

Figure 1. Expression of glutamate transporter-1 (GLT-1) and glutamine synthetase (GS) in the hippocampal CA1 area after reperfusion for 1, 3,5 and 7 days. (A) Compared with the ischemia group, Buyang Huanwu decoction (BYHWD) upregulated the expression of GLT-1 from day 1, and peaked on day 3 (magnification, $\mathrm{x} 400$ ). (B) BYHWD induced an increase in GS immunoreactivity (IR), particularly on day 3 (magnification, $\mathrm{x} 400$ ). ${ }^{* *} \mathrm{P}<0.01$ vs. ischemia group. ${ }^{\# \#} \mathrm{P}<0.01$ vs. ischemia + BYHWD group on days 1,5 and 7.

were presented as mean \pm standard error of the mean, and significant differences between groups were analyzed with one-way analysis of variance. The Student's t-test was used to compare the means of two samples. All the analysis was carried out using the Statistical Package for Social Sciences (SPSS, version 13.0 for Windows; SPSS, Inc., Chicago, IL, USA). $\mathrm{P}<0.05$ was considered to indicate a statistically significant difference.

\section{Results}

Elevation of GLT-1 and GS by BYHWD administration following focal ischemia. Expression of GLT-1 and GS was examined after ischemia for $2 \mathrm{~h}$ and reperfusion for 1, 3, 5 and 7 days, respectively, to observe their changes over time. Compared with the control group, ischemia significantly decreased GLT-1 expression. Among the ischemia group, GLT-1 expression in the hippocampus CA1 area on day 3 was higher than that on day 1 , and it subsequently decreased on days 5 and $7(\mathrm{~F}=123.014, \mathrm{P}<0.001)$. Additionally, BYHWD significantly enhanced GLT-1 expression compared with the ischemia group, with the distinct difference on day 3 $(\mathrm{F}=171.564, \mathrm{P}<0.001)$ (Fig. 1A).

Similar with GLT-1, BYHWD markedly upregulated the expression of GS in the hippocampal CA1 region from day 1 and peaked on day 3 compared with the ischemia group. The level of GS was gradually decreased, but a significant difference remained between the BYHWD and ischemia groups on days 5 and 7 ( $F=104.439, P<0.001)$ (Fig. 1B).

Alteration of PACAP-38 and its receptor antagonist downregulates the expression of GLT-1 and GS. As BYHWD upregulated the expression of GLT-1 and GS expression on day 3 , the following experiments were carried out on day 3. As shown in Fig. 2, focal ischemia for $2 \mathrm{~h}$ followed by $72 \mathrm{~h}$ of reperfusion resulted in less PACAP-38 expression $(\mathrm{t}=3.987, \mathrm{P}=0.017)$, but administration of BYHWD significantly increased the expression $(\mathrm{t}=3.999, \mathrm{P}=0.016)$ (Fig. 2A). Combined with the fact that PACAP-38 regulated glial glutamate transportation and metabolism, whether PACAP-38 was involved in the process of BYHWD protecting ischemia injury by enhancing GLT-1 and GS. As shown in Fig. 2, focal ischemia for $2 \mathrm{~h}$ followed by $72 \mathrm{~h}$ of reperfusion resulted in decreased GLT-1 $(\mathrm{t}=20.222, \mathrm{P}<0.001)$ and GS $(\mathrm{t}=14.596$, $\mathrm{P}<0.001)$. BYHWD enhanced GLT-1 $(\mathrm{t}=10.654, \mathrm{P}<0.001)$ and GS $(\mathrm{t}=11.794, \mathrm{P}<0.001)$ expression compared with the ischemia group, while co-administration of PACAP-(6-38) (a non-specific PACAP receptor antagonist) significantly reduced the expression of GLT-1 $(\mathrm{t}=9.798, \mathrm{P}<0.001)$ and $\mathrm{GS}(\mathrm{t}=5.368$, $\mathrm{P}=0.001$ ) (Fig. 2B-D). 

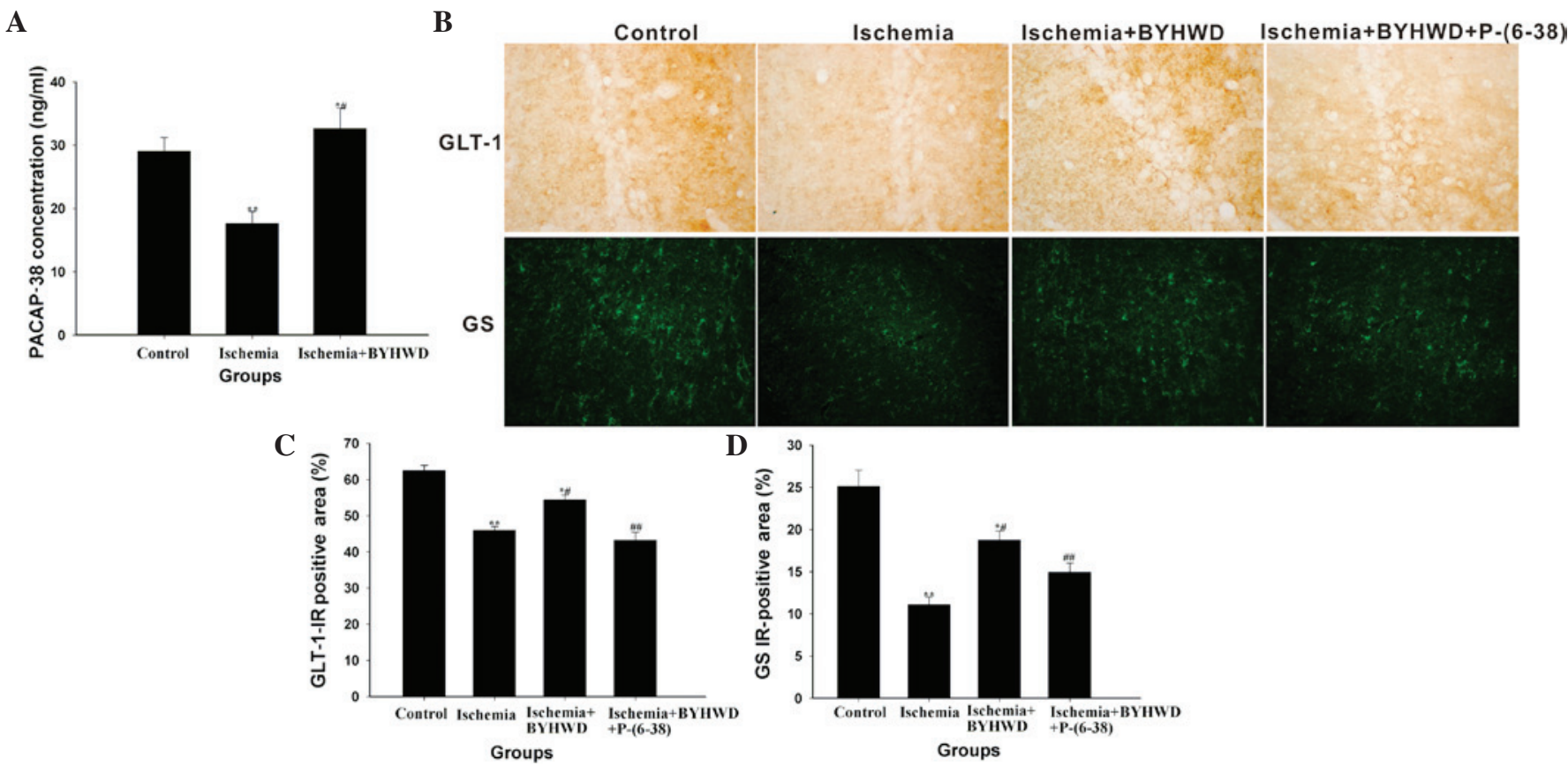

Figure 2. Pituitary adenylate cyclase activating polypeptide-38 (PACAP-38) played an important role in the upregulation of glutamate transporter-1 (GLT-1) and glutamine synthetase (GS) following Buyang Huanwu decoction (BYHWD) treatment. (A) Enzyme-linked immunosorbent assay for PACAP-38 showed that BYHWD significantly induced an increase in PACAP-38 in the hippocampus CA1 area. (B and C) Ischemia resulted in decreased GLT-1 expression, while BYHWD attenuated the decrease. Additionally, P-(6-38) (a non-specific PACAP antagonist) inhibited the effect of BYHWD (magnification, x400). (B and D) Ischemia led to decreased GS expression, while BYHWD attenuated the decrease and P-(6-38) blocked this effect (magnification, $\mathrm{x} 400)$. ${ }^{* *} \mathrm{P}<0.01$ vs. control group; ${ }^{* \#} \mathrm{P}<0.01$ vs. ischemia group; ${ }^{\# \#} \mathrm{P}<0.01$ vs. ischemia $+\mathrm{BYHWD}$ group.

A

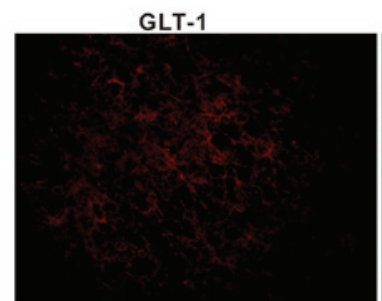

B

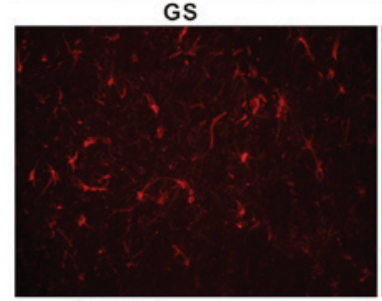

C

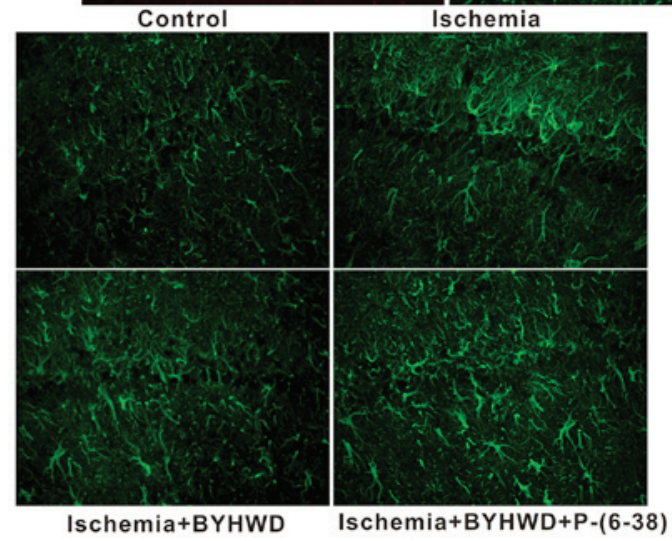

GFAP

GFAP


Figure 3. Glial fibrillary acidic protein (GFAP) was affected by Buyang Huanwu decoction (BYHWD). (A) Double immunofluorescence showed that glutamate transporter-1 (GLT-1) was mainly colocalized with GFAP (magnification, x400). (B) Double immunofluorescence showed that glutamine synthetase (GS) was mostly colocalized with GFAP (magnification, $\mathrm{x} 400$ ). (C and D) Ischemia resulted in increased GFAP expression, while BYHWD decreased its expression. Additionally, P-(6-38) inhibited the downregulation of GFAP by BYHWD (magnification, $\mathrm{x} 400$ ). ${ }^{* *} \mathrm{P}<0.01$ vs. control group; ${ }^{* *} \mathrm{P}<0.01 \mathrm{vs}$. ischemia group; ${ }^{\# \#} \mathrm{P}<0.01$ vs. ischemia + BYHWD group. 
Alteration of GFAP byBYHWD. As shownin Fig.3,GLT-1 andGS were mainly colocalized with GFAP (an indicator of astrocytes) detected by double immunofluorescent staining (Fig. 3A and B), indicating that they were mainly expressed in astrocytes. Subsequently, whether BYHWD affected GFAP immunostaining was investigated. Immunofluorescent staining showed that GFAP expression was enhanced in the ischemia group compared with the control group $(\mathrm{t}=10.579, \mathrm{P}<0.001)$. However, BYHWD significantly attenuated the expression $(\mathrm{t}=6.481$, $\mathrm{P}<0.001)$. Additionally, co-administration of PACAP-(6-38) reversed the effect of BYHWD on decreasing GFAP expression ( $\mathrm{t}=5.418, \mathrm{P}=0.001$ ) (Fig. 3C and $\mathrm{D}$ ). Representative images showed that astrocytes in hippocampal CA1 appeared to exhibit hypertrophic morphology in the ischemia group, while BYHWD attenuated the astrogliosis, and PACAP-(6-38) inhibited the effect of BYHWD (Fig. 3C).

\section{Discussion}

It is well known that during ischemia, elevated glutamate leads to a rise in the intracellular sodium and calcium, which would further result in mitochondrial dysfunction, activation of proteases, accumulation of reactive oxygen species and release of nitric oxide. All these substances contribute to ischemic injury. GLT-1, which is responsible for the majority of glutamate transportation, plays a critical role in preventing overstimulation of glutamate receptors. Ischemia preconditioning and certain agents, such as ceftriaxone, were reported to protect ischemic injury through upregulating GLT-1 (21). Additionally, the metabolic conversion of glutamate to glutamine by GS could accelerate the extracellular glutamate uptake by reducing the intracellular glutamate concentration, thus playing a protective role following ischemia (22). In the present study, the levels of GLT-1 and GS increased on day 3 in the ischemia group, indicating that an internal potential protective mechanism within the body is functioning. BYHWD augmented this effect, which was in accordance with the theory of Chinese Medicine and provided more evidence for elucidating its mechanisms of anti-cerebral ischemia. However, certain investigators have reported that the effect of BYHWD on decreasing glutamate peaked at $3 \mathrm{~h}$ after administration $(6,7)$, but there should be other mechanisms, as BYHWD absorption and changes of protein expression possibly require a longer period of time. Although upregulation of GLT-1 and GS in the present study did not appear earlier to prevent ischemic injury efficiently, they could be beneficial for subsequent strokes, later regeneration and functional restoration, as GLT-1 was reported to be involved in synaptic plasticity and associative fear memory (23).

Recently, PACAP-38, which reduced infarct volume and attenuated the neuronal injury (16), has been found to be involved in GLT-1 and GS expression. Exposure of cultured astroglia to PACAP-38 increased the maximal velocity of glutamate uptake by promoting the expression of GLT-1 and GS. The stimulatory effects of neuron-conditioned medium on GLT-1 and GS were attenuated in the presence of PACAP receptor antagonist PACAP-(6-38) (15). The present data showed that PACAP-38 expression was increased following BYHWD administration. In addition, PACAP-(6-38) attenuated the effect of BYHWD on GLT-1 and GS. These results indicated that PACAP-38 played an important role in the effect of BYHWD on GLT-1 and GS. By contrast, the usage of PACAP-(6-38) also well-illustrated the specific effects of BYHWD on GLT-1 and GS to a certain extent.

Of note, BYHWD upregulated GLT-1 and GS, and decreased GFAP expression in the present study. It appears that the GFAP level was conversely associated with the expression of GLT-1 and GS, which is consistent with previous results. For example, ceftriaxone, which upregulated GLT-1 expression, blocked elevated GFAP expression $(24,25)$. By contrast, downregulation of GLT-1 and GS occurred in activated astrocytes characteristic of increased GFAP expression (26). The present results further verified the antagonism between the expression of GLT-1 and GS and glial cell proliferation. Notably, although astrocytes have already been recognized to play critical roles following ischemia $(27,28)$, few studies explored the role of astrocytes in BYHWD protecting cerebral ischemia. The present study demonstrated that the protective function of BYHWD was, in part through upregulating GLT-1 and GS in astrocytes, providing a strong evidence to elucidate the role of astrocytes in BYHWD protective effects following ischemia.

Taken together, BYHWD treatment for focal ischemia induced a significant increase of GLT-1 and GS expression. PACAP-(6-38) significantly inhibited this effect, suggesting that PACAP-38 contributed to the effect of BYHWD on GLT-1 and GS in astrocytes. These findings not only answer how BYHWD decreased glutamate concentration, but also provide direct evidence that astrocytes closely participated in the protective role of BYHWD following focal ischemia, which may be a new point for the study of traditional medicine. In addition, impaired glutamate transport is known to induce neurotoxicity associated with numerous neurological processes, such as Alzheimer's disease, Huntington's disease and amyotrophic lateral sclerosis $(29,30)$. This highlights the importance of the BYHWD treatment not only in stroke outcome, the main focus of the present study, but also in other neuropathologies, which deserve further study.

\section{Acknowledgements}

The present study was supported by the National Nature Science Foundation (grant no. 81202817), the Guangdong Province Outstanding Young Teachers in Higher Education Institutions (grant no. Yq2013041), the Natural Science Foundation of Guangdong Province (grant no. S2012040007519) and the Guangdong College of Outstanding Youth Innovation Talent Training Project (grant nos. LYM11042 and 2012LYM0045).

\section{References}

1. Wei RL, Teng HJ, Yin B, Xu Y, Du Y, He FP, Chu KT, Luo BY and Zheng GQ: A systematic review and meta-analysis of buyang huanwu decoction in animal model of focal cerebral ischemia. Evid Based Complement Alternat Med 2013: 138484, 2013.

2. Wang L and Jiang DM: Neuroprotective effect of Buyang Huanwu Decoction on spinal ischemia-reperfusion injury in rats is linked with inhibition of cyclin-dependent kinase 5. BMC Complement Altern Med 13: 309-315, 2013.

3. Li XM, Bai XC, Qin LN, Huang H, Xiao ZJ and Gao TM: Neuroprotective effects of Buyang Huanwu Decoction on neuronal injury in hippocampus after transient forebrain ischemia in rats. Neurosci Lett 346: 29-32, 2003. 
4. Yang S, Gao Q, Xing S, Feng X, Peng L, Dong H, Bao L, Zhang J, $\mathrm{Hu}$ Y, Li G, et al: Neuroprotective effects of Buyang Huanwu decoction against hydrogen peroxide induced oxidative injury in Schwann cells. J Ethnopharmacol 137: 1095-1101, 2011.

5. Cai G, Liu B, Liu W, Tan X, Rong J, Chen X, Tong L and Shen J: Buyang Huanwu Decoction can improve recovery of neurological function, reduce infarction volume, stimulate neural proliferation and modulate VEGF and Flk1 expressions in transient focal cerebral ischaemic rat brains. J Ethnopharmacol 113: 292-299, 2007.

6. Zhao LD, Wang JH, Jin GR, Zhao $Y$ and Zhang $H J$ Neuroprotective effect of Buyang Huanwu decoction against focal cerebral ischemia/reperfusion injury in rats - time window and mechanism. J Ethnopharmacol 140: 339-344, 2012.

7. Wang L, Huang Y, Wu J, Lv G, Zhou L and Jia J: Effect of Buyang Huanwu decoction on amino acid content in cerebrospinal fluid of rats during ischemic/reperfusion injury. J Pharm Biomed Anal 86: 143-150, 2013.

8. Kostandy BB: The role of glutamate in neuronal ischemic injury: The role of spark in fire. Neurol Sci 33: 223-237, 2012.

9. Robinson MB: The family of sodium-dependent glutamate transporters: A focus on the GLT-1/EAAT2 subtype. Neurochem Int 33: 479-491, 1998.

10. Gong SJ, Chen LY, Zhang M, Gong JX, Ma YX, Zhang JM, Wang YJ, Hu YY, Sun XC, Li WB, et al: Intermittent hypobaric hypoxia preconditioning induced brain ischemic tolerance by up-regulating glial glutamate transporter-1 in rats. Neurochem Res 37: 527-537, 2012

11. Zhang W, Miao Y,Zhou S, Wang B, Luo Q and Qiu Y: Involvement of glutamate transporter-1 in neuroprotection against global brain ischemia-reperfusion injury induced by postconditioning in rats. Int J Mol Sci 11: 4407-4416, 2010.

12. Rothstein JD, Patel S, Regan MR, Haenggeli C, Huang YH, Bergles DE, Jin L, Dykes Hoberg M, Vidensky S, Chung DS, et al: Beta-lactam antibiotics offer neuroprotection by increasing glutamate transporter expression. Nature 433: 73-77, 2005.

13. Zou J, Wang YX, Dou FF, Lü HZ, Ma ZW, Lu PH and Xu XM: Glutamine synthetase down-regulation reduces astrocyte protection against glutamate excitotoxicity to neurons. Neurochem Int 56: 577-584, 2010.

14. Lee A, Lingwood BE, Bjorkman ST, Miller SM, Poronnik P, Barnett NL, Colditz P and Pow DV: Rapid loss of glutamine synthetase from astrocytes in response to hypoxia: Implications for excitotoxicity. J Chem Neuroanat 39: 211-220, 2010.

15. Figiel $\mathrm{M}$ and Engele J: Pituitary adenylate cyclase-activating polypeptide (PACAP), a neuron-derived peptide regulating glial glutamate transport and metabolism. J Neurosci 20: 3596-3605, 2000.

16. Reglodi D, Somogyvari-Vigh A, Vigh S, Maderdrut JL and Arimura A: Neuroprotective effects of PACAP38 in a rat model of transient focal ischemia under various experimental conditions. Ann NY Acad Sci 921: 119-128, 2000.

17. Chen A, Wang H, Zhang J, Wu X, Liao J, Li H, Cai W, Luo $\mathrm{X}$ and Ju G: BYHWD rescues axotomized neurons and promotes functional recovery after spinal cord injury in rats. J Ethnopharmacol 117: 451-456, 2008.
18. Ding W, Zhou L, Liu W, Guan L, Li X, Liu H, Yan F, Xu J, Zeng W and Qiu M: Opposite effects of the gap junction blocker octanol on focal cerebral ischemia occluded for different durations. Mol Med Rep 9: 2485-2490, 2014

19. Vaudry D, Gonzalez BJ, Basille M, Fournier A and Vaudry H: Neurotrophic activity of pituitary adenylate cyclase-activating polypeptide on rat cerebellar cortex during development. Proc Natl Acad Sci USA 96: 9415-9420, 1999.

20. Chen YD, Zhou ZG, Wang Z, Gao HK, Yan WW, Wang C, Zhao GP and Peng XH: Pituitary adenylate cyclase activating-peptide and its receptor antagonists in development of acute pancreatitis in rats. World J Gastroenterol 11: 538-544, 2005.

21. Lee SG, Su ZZ, Emdad L, Gupta P, Sarkar D, Borjabad A, Volsky DJ and Fisher PB: Mechanism of ceftriaxone induction of excitatory amino acid transporter-2 expression and glutamate uptake in primary human astrocytes. J Biol Chem 283: 13116-13123, 2008

22. Lo JC, Huang WC, Chou YC, Tseng CH, Lee WL and Sun SH: Activation of $\mathrm{P} 2 \mathrm{X}(7)$ receptors decreases glutamate uptake and glutamine synthetase activity in RBA-2 astrocytes via distinct mechanisms. J Neurochem 105: 151-164, 2008.

23. Li YK, Wang F, Wang W, Luo Y, Wu PF, Xiao JL, Hu ZL, Jin Y, $\mathrm{Hu} \mathrm{G}$ and Chen JG: Aquaporin-4 deficiency impairs synaptic plasticity and associative fear memory in the lateral amygdala: Involvement of downregulation of glutamate transporter-1 expression. Neuropsychopharmacology 37: 1867-1878, 2012.

24. Ramos KM, Lewis MT, Morgan KN, Crysdale NY, Kroll JL, Taylor FR, Harrison JA, Sloane EM, Maier SF and Watkins LR: Spinal upregulation of glutamate transporter GLT-1 by ceftriaxone: Therapeutic efficacy in a range of experimental nervous system disorders. Neuroscience 169: 1888-1900, 2010.

25. Goodrich GS, Kabakov AY, Hameed MQ, Dhamne SC, Rosenberg PA and Rotenberg A: Ceftriaxone treatment after traumatic brain injury restores expression of the glutamate transporter, GLT-1, reduces regional gliosis, and reduces post-traumatic seizures in the rat. J Neurotrauma 30: 1434-1441, 2013.

26. Kruchkova Y, Ben-Dror I, Herschkovitz A, David M, Yayon A and Vardimon L: Basic fibroblast growth factor: A potential inhibitor of glutamine synthetase expression in injured neural tissue. J Neurochem 77: 1641-1649, 2001.

27. Rossi DJ, Brady JD and Mohr C: Astrocyte metabolism and signaling during brain ischemia. Nat Neurosci 10: 1377-1386, 2007.

28. Zhao Y and Rempe DA: Targeting astrocytes for stroke therapy. Neurotherapeutics 7: 439-451, 2010

29. Gegelashvili G, Robinson MB, Trotti D and Rauen T: Regulation of glutamate transporters in health and disease. Prog Brain Res 132: 267-286, 2001

30. Trotti D, Aoki M, Pasinelli P, Berger UV, Danbolt NC, Brown RH Jr and Hediger MA: Amyotrophic lateral sclerosis-linked glutamate transporter mutant has impaired glutamate clearance capacity. J Biol Chem 276: 576-582, 2001. 